\title{
Application of sustained release microsphere in hypoparathyroidism after thyroid surgery
}

\author{
Ming Pan, Hong Zhang ${ }^{1}$ and Yueshu Wang ${ }^{2}$ a \\ ${ }^{1}$ Department of thyroid Surgery, the Second Affiliated Hospital of Jilin University, 130041, Changchun, China \\ ${ }^{2}$ Department of hand surgery, China-Japan Union Hospital of Jilin University, 130033, Changchun, China
}

\begin{abstract}
Hypoparathyroidism (HypoPT) is one of the most common complications after thyroid surgery. The parathyroid hormone-controlled delivery system is one of the most significant therapies for hypothyroidism after thyroid and parathyroid surgery. The character of biodegradability and the slow release of PTH of the Sustained release microspheres were widely concerned. The authors present the research progress in sustained release microspheres loaded with PTH from the character, material, fabrication and application.
\end{abstract}

\section{Introduction}

Hypoparathyroidism (HypoPT) is diagnosed on the foundation of hypocalcemia and low plasma levels of parathyroid hormone (PTH). Recent studies from the USA and other countries have estimated that the prevalence of HypoPT is 25 per 100,000 individuals[1-3].Risk factors for permanent hypothyroidism include seropositivity for thyroid peroxidase(TPO) antibodies, high normal preoperative thyroid stimulating hormone(TSH) levels, and histologically confirmed thyroiditis, but not age, sex, family history, or weight of the resected tissue. In most instances, HypoPT is due to accidental damages to or removal of the parathyroid glands during thyroid and parathyroid surgery. Permanent HypoPT arises not only after total or subtotal thyroidectomy, but also in $11 \%$ to $28 \%$ of patients that have undergone hemithyroidectomy[4-6].Hormone replacement after thyroid and parathyroid surgery is a common and significant clinical therapy. The parathyroid Sustained release microsphere is one of the most significant therapies for hypothyroidism after thyroid and parathyroid surgery. Controlled release technology enables the delivery of a biologically active agent at specific times with defined concentrations by encapsulating the biologically active agent within a biodegradable polymer in the form of a microsphere.

\section{Sustained release microsphere}

\subsection{Character}

The material of microspheres should meet the following requirements[7]:

1)Satisfactory biocompatibility, including blood compatibility, histocompatibility and nonimmunogenicit. Moreover, non-poisonous and non- teratogenic, Products after degradation have no

\footnotetext{
${ }^{a}$ Corresponding author:ysw_1971@163.com
} 
toxic action, won't cause inflammation and jump reaction.

2)Wonderful biodegradability, and degradation time and drug release rate can be regulated and controled as requestment.

3)The degradation of polymer must be in a suitable time.

4)The material should possess an applicable mechanical strength, and can be shaped through conventional method.

5)The method is simple.

6)Material sourcesis is easy to obtained, and it is inexpensive and suitable for large scale application. The deactivation of protein drugs must be avoided if it is used to load protein drugs.

\subsection{Material}

There are many biodegradable polymer materials used for embedding proteins, peptides, nucleic acids, vaccine and other active substances or other drugs. According to the origin, it can be divided into natural polymer materials, semisynthetic polymer materials and synthetic polymer materials. The characteristics of natural polymer materials are extensive sources, low cost, stable character, non-toxic, biodegradable, prominent film-forming and ballability, such as gelatin, alginate, chitosan, etc[89].Semisynthetic polymer materials are mostly cellulose derivatives. They have low virulence high viscosity, increased solubility after salt [10-11].Compared with natural materials, synthetic polymer materials has rich material source, and the structure and properties can be artificially modified and regulation. Over the past 20 years these materials developed rapidly, Polylactic acid and polyglycolic acid are the most typical synthetic biodegradable polymers, with non-toxic, no immune stimulation and superior biodegradability and biocompatibility[12-13].FDA approval polylactic acid glycolic acid (PLGA) as a biodegradable materials used in pharmaceutical excipients, PLGA has become the most widely applied biodegradable polymer materials.[14-15]So far, FDA has approved eight kinds of injectable microsphere drugs. All the microsphere materials are PLGA.

\subsection{Fabrication}

Presently, the preparation method of polymer microspheres includes spray drying, coacervation, multi ple emulsion-solvent evaporation and microporous membrane emulsification technique. The protein $\mathrm{d}$ rugs need to imbed into polymer materials should be dissolved uniformly in the water, and PLGA sho uld be dissolved in organic solvent, producing $\mathrm{W} / \mathrm{O} / \mathrm{W}$ compound emulsion, then reach a desiccation $\mathrm{b}$ y spraying in inert air, with the solvent evaporated quickly, embedding the drugs[16-17].Condensation is also called phase separation, Drugs and materials appear two phase separation after drugs and poly mer materials mixed each other under a certain external factors stimulation, Carrier polymer materials dissolve out from solution packaging drugs. Multiple emulsion-solvent evaporation is a process that a queous solution of drug and dichloromethane solution dissolved with PLGA are mixed, following wit $\mathrm{h}$ fabricate primal emulsion by ultrasound and other ways. Then the primal emulsion are infused in po ur water becoming $\mathrm{W} / \mathrm{O} / \mathrm{W}$ multiple emulsion-solvent. At last, to PLGA microspheres were fabricated by a solvent evaporation technique, embedding drugs[18]. Microporous membrane emulsification tec hnique[19] is a new technology developed from emulsion preparation. Disperse phase were pressed in fenestra under nitrogen pressure. When the forming emulsion drops reach a certain size at the fenestra exit, they were divorced from the surface of emulsion under the action of forces, entering into continu ous phase.

\subsection{Defect}

The size was not uniform, which is not only poor repeatability in preparation that make the process more complex, but also lead to inconsistent drug release behavior, causing unsteady blood drug concentration of patients. [20] The energy consumption of spray drying process is high that is easy to cause the inactivation of protein drugs, reducing the effect. Condensation need more organic solvents, 
which is difficult to wipe off the residual organic reagents. It is easier to become agglomerate by phase separation and more difficult to conduct mass production.

\section{Application of Sustained release microsphere loaded with PTH}

\subsection{Hormone replacement therapy}

Microspheres can be used to provide a bioactive calcium sensitivity control $\mathrm{PTH}$, and provides a potential way in hormone replacement therapy. Biodegradable poly(lactide-co-glycolide) (PLGA) microspheres loaded with PTH were made by Christopher Breuer[21] using a modification of the double emulsion (water/oil/water) solvent evaporation technique. Controlled release of PTH was demonstrated in vitro over a 3-week period and release of physiological significant concentrations of PTH was achieved using this methodology. In 2007,Christopher K.Breuer[22] used the spontaneous emulsion technique and fabricated PTH microspheres by encapsulating PTH(1-34) in PLGA. Another kind of microspheres were generated by mixing calmodulin with $\mathrm{PTH}(1-34)$ in the PLGA microspheres. It caused a significant increase in serum calcium levels when PTH released from the $\mathrm{PTH} /$ calmodulin microspheres.

\subsection{The growth and repairment of bone}

The developed PLGA microspheres with sustained release and long-term effect show great potential as a promising delivering and releasing carriers for inducing a biologically active response in bone, treating early osteoarthritis and osteoporosis. Rajalakshmanan Eswaramoorthy[23] developed an effective controlled-release system for prolonging the treatment duration of an intra-articular injection for osteoarthritis treatment in rats. The effect of PTH/PLGA microspheres on suppressing osteoarthritis progression was similar to that of a once-every-three-day injection of PTH(1-34), indicating that both the sustained and intermittent action of $\mathrm{PTH}(1-34)$ effectively suppress osteoarthritis progression. In the same year, Peter X. Ma[24] prepared poly(lactic-co-glycolic acid) (PLGA) microspheres containing bovine serum albumin (BSA) or human PTH1-34 by using a double emulsion method with high encapsulation efficiency and controlled particle sizes. And both in vitro and in vivo assays demonstrated that the bioactivity of PTH was maintained largely during the fabrication of PLGA microspheres and upon release. Recently, in Wei-yan Meng[25] prepared PLGA microspheres by a water-in-oil-in-water double emulsion solvent evaporation and PTH1-34 was encapsulated to the microspheres by a technique with good sphericity and high efficiency. The polyester microspheres had a good spherical shape.PTH1-34 loaded microspheres exhibited a slow release behavior for about 25 days. Cell proliferation experiments demonstrated that PTH1-34 loaded PLGA microspheres can enhance the proliferation and differentiation of MC3T3 cell effectively. The cells incubated with PTH1-34 loaded PLGA microspheres exhibited higher ALP level.

\section{Conclusion and expectation}

Sustained release microspheres are still in the laboratory research stage, with a lot of problems need to be solved. However, the character of biodegradability and the slow release of PTH of the Sustained release microspheres were widely concerned. It is a hopeful role in physiologically controlled drugdelivery way of PTH in clinical practice, especially, in the therapy of hypoparathyroidism after thyroid surgery.

\section{References}

1. L. Underbjerg, T. Sikjaer, L. Mosekilde, L. Rejnmark. J Bone Miner Res.28,2277-2285(2013).

2. J. Powers, K. Joy, A. Ruscio, H. Lagast. J Bone Miner Res,28,2570-2576(2013). 
3. L. Underbjerg, T. Sikjaer, L. Mosekilde, L. Rejnmark. J Bone Miner Res,30,1738-44(2015).

4. R. Gärtner, M. Reincke. Der Internist.49,538-544(2008).

5. A. Schäffler, Diabetologie und Stoffwechsel. 36(2009).

6. W.M. Wiersinga. Horm Res, 56(Suppl 1), 74-81(2001).

7. A. Kumari, S.K. Yadav, S.C. Yadav. Colloid Surfaces B, 75(1),1-18(2010).

8. M.S. Soliman, T. Behzad. Pharm Dev Tech, 2(3), 33-35(2014).

9. X Hua,S Tan,HM Bandara,Y Fu,S Liu.Plos One, 9(12),e114271(2014).

10. S. Butu,F.G. Ince,H. Erdugan, N. Sahiner. Carbohydrate Polymers, 86(2), 636-643(2011).

11. J. Shaji,V. Patole. Indian J Pharm Sci, 70(3), 269-277(2008).

12. V. Lassall,M.L. Ferreira. Macromol Biosci, 7( 6),767-783(2007).

13. J.A. Floyd, A. Galperin, D.R. Buddy. Adv Drug Deliver Rev, 91,23-37(2015).

14. K.M. Hirenkuma, J.S. Steven. Polymers, 3(3), 1377-1397(2011).

15. G. Orive, A.R. Gascón, R.M. Hernández, A. Domínguez-Gil,J.L. Pedraz. Trends Pharmacol Sci, 25(7), 382-387(2004).

16. T. Petreska-Ivanovska,L. Petrushevska-Tozi,A. Grozdanov,R. Petkovska,J. Hadjieva. Chem Ind Chem Eng Q, 20 (4),549-564(2014).

17. F. Sansone, T. Mencherini, P. Picerno, T. Esposito, P.D. Gaudio. J Pharm pharmacol, 2 (4),100109(2014).

18. J. Naik, R.D. Kulkarni, A.B. Lokhande, S. Mishra. Int J Pharm Bio Sci, 3(4),573 -590(2012).

19. C. Charcosset, I. Limayem, H. Fessi. J Chem Technol Biot, 79(3), 209-218(2004).

20. L.Y. Wang,G.H. Ma,Z.G. Su. J Controlled Release, 106(1-2), 62-75(2005).

21. T. Anthony,P. Fong,A. Goyal,W.M._Saltzman,R.L. Moss. Journal of Pediatric Surgery, 40(1),81$85(2005)$.

22. P. Fong, A. Goyal, M. Brennan, J. Park, L. Moss. Journal of Surgical Research, 143(2),195199( 2008).

23. R. Eswaramoorthy, C.C. Chang, S.C. Wu, G.J. Wang, J.K. Chang. Acta Biomaterialia, 8(6),22542262(2012).

24. G. Wei, G.J. Pettway, L.K. Mccauley, P.X. Ma. Biomaterials, 25(2):345-352(2004).

25. S.B. Zhang, Z. Zhang, M.N. Yu, T.B. Liu, J.J. Wang. Acta Polymerica Sinica, 014(2),270275(2014). 